\title{
ARC is highly expressed in nasopharyngeal carcinoma and confers $X$-radiation and cisplatin resistance
}

\author{
PING WU, YAOYUN TANG, JIAN HE, LIN QI, WEIHONG JIANG and SUPING ZHAO \\ Department of Otolaryngology, Xiangya Hospital, Central South University, Changsha, Hunan 410008, P.R. China
}

Received May 13, 2013; Accepted June 12, 2013

DOI: $10.3892 /$ or.2013.2622

\begin{abstract}
Apoptosis repressor with caspase recruitment domain (ARC), an inhibitor of apoptosis, is primarily expressed in terminally differentiated tissues. Recent studies have revealed that ARC is highly expressed in a variety of human cancer cell lines and epithelial-derived cancers, which suggests that ARC plays an important role in the process of carcinogenesis. However, whether ARC is involved in the development of nasopharyngeal carcinoma (NPC) and the various roles it plays in NPC remain unclear. In the present study, we examined the expression of ARC in NPC cell lines and NPC tissues and the relationship between its subcellular expression and clinicopathological grade; moreover, we explored the effect of this protein on radiation resistance and chemoresistance in NPC cells. We found that cytoplasmic ARC was expressed at high levels in NPC tissues, at moderate levels in severe atypical hyperplasia and at low levels in benign nasopharyngeal tissues. High expression of cytoplasmic and nuclear ARC was correlated with advanced local invasion. However, only a small amount of nuclear ARC was expressed in NPC in contrast to cytoplasmic ARC. We also found that attenuation of ARC expression by miRNA resulted in decreased X-radiation and cisplatin resistance in NPC CNE-2 cells. In contrast, overexpression of $\mathrm{ARC}$ resulted in increased X-radiation and cisplatin resistance in NPC 6-10B cells. Furthermore, we demonstrated that ARC appears to be critical for blocking the activation of casapse- 8 and casapse- 2 in NPC cells subjected to X-radiation or cisplatin. These results suggest that high expression of ARC plays an important role in the pathogenesis of NPC and leads to $\mathrm{X}$-radiation and cisplatin resistance in NPC.
\end{abstract}

\section{Introduction}

Apoptosis is a form of procedural cell death. It plays a role in tissue growth and dynamic equilibrium, as well as in numerous

Correspondence to: Professor Suping Zhao, Department of Otolaryngology, Xiangya Hospital, Central South University, Xiangya Road 87, Changsha, Hunan 410008, P.R. China

E-mail: xyxzsp@126.com

Key words: apoptosis repressor with caspase recruitment domain, nasopharyngeal carcinoma, apoptosis, radiation, cisplatin diseases, including cancer, autoimmune diseases, viral infections and degenerative diseases $(1,2)$. Apoptosis is mediated by two integrated pathways, the extrinsic and intrinsic apoptotic pathways. In the extrinsic pathway, the combination of ligands and cell surface death receptors stimulates formation of the death-inducing signaling complex (DISC), which further activates upstream caspase- 8 . Activated caspase- 8 stimulates downstream caspase molecules, inducing hydrolysis of a large number of cellular proteins and eventually leads to cell death. In the intrinsic pathway, a stimulus signal is delivered to the mitochondria and endoplasmic reticulum (ER) through the interaction of BH3-only and Bcl-2 family proteins, which in turn triggers the release of apoptogen cytochrome $c$ from mitochondria to the cytoplasm. The cytochrome $c$ in the cytoplasm interplays with apoptosis-related factor 1 (Apaf-1) in the presence of dATP and stimulates formation of apoptosomes and activation of upstream caspase-9, which subsequently activates downstream caspases, finally inducing apoptosis. Therefore, the activation of the apoptotic pathway eventually leads to mitochondrial dysfunction, caspase activation and cell death.

Although these pathways are mediated by death signal stimulation and activation of various pro-apoptotic factors, each stage of the apoptotic pathway is regulated at additional levels by endogenous proteins that play a role in reverse adjustment. These proteins include FLIP, which opposes the extrinsic pathway; Bcl-2 and Bcl-xL, which block the release of apoptogens from the mitochondria; and IAPs, which inhibit the activation of downstream caspases.

ARC (apoptosis repressor with caspase recruitment domain) is an apoptotic inhibitor with a caspase enrichment of functional domains. It is an endogenous CARD containing protein that inhibits apoptosis by selectively binding to and inhibiting caspase- 8 and caspase-2, but not caspase-1, caspase- 9 and caspase-3 $(3,4)$. It can also directly combine with FADD and Fas, and block the interaction of Fas and FADD, thereby inhibiting the assembly of DISC (4). In addition, interactions between ARC and pro-apoptotic factor Bax preclude activation of Bax and its translocation to the mitochondria (5). In addition, the proline-glutamic acid-riched region of ARC can be directly bound to the tetramerization domain of p53, thereby blocking p53 tetramerization and suppressing p53 transfer from the cytoplasm to the nucleus (6). Actually, ARC can inhibit apoptosis induced by death receptors, hypoxia, oxidative stress, serum deprivation and ischemia-reperfusion $(3,4,7-9)$. 
As inhibition of apoptosis has been implicated in carcinogenesis, ARC potently inhibits a wide array of death signals, which suggests that it may play a functionally important role in the process of carcinogenesis. It remains unknown whether ARC plays a role in the development of nasopharyngeal carcinoma (NPC). In the present study, we assessed the abundance of ARC in NPC cell lines and NPC tissues and the relationship between its expression and clinicopathological tumor grade. Moreover, we sought to assess the effects of ARC expression, through downregulation and overexpression of ARC, on the response of NPC cell lines with diverse intrinsic radiation- and chemo-sensitivities and different levels of ARC expression.

\section{Materials and methods}

Materials. Human NPC cell lines CNE-1, CNE-2, 5-8F, 6-10B and HNE-2 have been previously described $(10,11)$. Formalinfixed, paraffin-embedded tissues and clinicopathological parameters were obtained from 82 patients with a pathological diagnosis of poorly differentiated squamous cell carcinoma, from 14 patients with severe atypical hyperplasia and from 20 patients with benign nasopharyngeal diseases at the Department of Otolaryngology and Pathology of the Xiangya Hospital. The present study was approved by the Ethics Committee of the Xiangya School of Medicine, Central South University, China.

Cell culture. NPC cells were cultured in RMPI-1640 medium (Gibco, Grand Island, NY, USA) supplemented with $10 \%$ fetal bovine serum (FBS; Gibco) at $37^{\circ} \mathrm{C}$ in an incubator with a humidified atmosphere with $5 \% \mathrm{CO}_{2}$ in air.

Immunohistochemical staining and counting methods. Immunohistochemical staining and counting methods were carried out as previously described $(10,11)$. Rabbit anti-human antibody against ARC (1:1,000 dilution; Abcam) was used and its expression was detected with biotinylated goat antirabbit IgG (1:1,000 dilution; Changxing Zhongshan Chemical Industry Co., Ltd., Changxing, China). 3',3'-Diaminobenzidine (Fuzhou Maixin, Fuzhou, China) was used for chromogenesis. Sections were blindly evaluated by two pathologists using light microscopy. Both the intensity and the percentage of positive cells were evaluated.

Western blotting. Western blotting was used as previously described $(10,11)$. A rabbit anti-human antibody against ARC (1:2,000 dilution) and mouse anti-human antibodies against caspase-8, caspase- 2 and $\beta$-actin (Santa Cruz Biotechnology, SantaCruz,CA,USA) wereused and theirexpression was detected with goat anti-rabbit or goat anti-mouse horseradish peroxidase (HRP) as secondary antibody (Beyotime Biotechnology, Beijing, China), respectively. Chemiluminescence reagent (Fermentas Biology) was used for enhanced electrochemiluminescence detection. The signal intensity was analyzed using GeneTools software (Syngene, Frederick, MD, USA). $\beta$-actin was used as a loading control.

RNA interference and plasmid transfection. microRNA (miRNA) against ARC and negative control miRNA (designed and synthesized by Invitrogen Biotechnology) were used for gene knockdown. The double-stranded oligo (top strand: 5'-TGCTGTTCTGCTTCAGCCTCGGGTTCGTTTTGGCC ACTGACTGACGAACCCGACTGAAGCAGAA-3'; bottom strand: 5'-CCTGTTCTGCTTCAGTCGGGTTCGTCAGTCA GTGGCCAAAACGAACCCGAGGCTGAAGCAGAAC-3') encoding the pre-miRNA for ARC was inserted into the miRNA expression vector pcDNA ${ }^{\mathrm{TM}} 6.2-\mathrm{GW} / \mathrm{EmGFP}-\mathrm{miR}$ (Invitrogen, Carlsbad, CA, USA) for construction of the expression plasmid. Expression of the plasmid was confirmed by DNA sequencing. Cells at $60-80 \%$ confluence were transfected with the miRNA plasmid at a final concentration of $100 \mathrm{nM}$ of the respective miRNA (ARC or control miRNA) using Lipofectamine ${ }^{\mathrm{TM}} 2000$ transfection reagent (Invitrogen) according to the manufacturer's instructions. After 14 days of selection in RPMI-1640 containing 10\% FBS and $10 \mu \mathrm{g} / \mathrm{ml}$ puromycin (Invitrogen), individual puromycin-resistant colonies were isolated and expanded. The ARC plasmid and control plasmids (EX-Z9301-M03, purchased from GeneCopoeia $\left.^{\mathrm{TM}}\right)$ were used for gene expression. Cells at $60-80 \%$ confluence were transfected with $1.6 \mu \mathrm{g} / \mathrm{ml}$ of the plasmid using Lipofectamine 2000 according to the manufacturer's instructions. After no less than 14 days of selection in RPMI-1640 containing 10\% FBS and neomycin $(300 \mu \mathrm{g} / \mathrm{ml}$; Santa Cruz Biotechnology), individual neomycin-resistant colonies were isolated and expanded. The expression of ARC was determined by western blot analysis.

Cell viability assay. Cell viability was detected using 2-(2-methoxy-4-nitrophenyl)-3-(4-nitrophenyl)-5-(2,4-disulfo phenyl)-2H-tetrazolium, monosodium salt (CCK-8 Kit; Beyotime). CCK-8 solution (10 $\mu \mathrm{l}$ ) was added to each well of 96-well plates with the same amount of culture fluid and drugs. CCK-8 solution without cell as well as blank plus. Optical densities were determined on a microtiter plate reader at $450 \mathrm{~nm}$.

Cell apoptosis. Annexin V-FITC apoptosis detection kit (Beyotime) was used to evaluate cell apoptosis. As previously described (10), cells were stained using Annexin-V-FITC for $10 \mathrm{~min}$ at room temperature and were then stained using propidium iodide (PI) for $10 \mathrm{~min}$ in the dark. The cells were analyzed immediately on a FACScan flow cytometer (BD Biosciences, Franklin Lakes, NJ, USA).

Radiation and drug treatment. Cells were seeded in triplicate in 96-well plates at a density of 3,000 cells for CNE-2 and 2,000 cells for $6-10 \mathrm{~B}$ per well in $100 \mu \mathrm{l}$ of the corresponding medium. Cells were exposed to the indicated X-ray irradiation doses (2, 4, 6, 8 and $10 \mathrm{~Gy})$. After $96 \mathrm{~h}$, viable cells were detected using the CCK- 8 assay kit. For drug treatment, cells were seeded in triplicate in 96-well plates at a density of 7,000 cells for CNE-2 and 3,000 cells for 6-10B per well in $100 \mu 1$ of the corresponding medium. On the following day, the media were discarded and replaced with fresh media containing the indicated concentrations of the following drug: cisplatin $(0,5$, $10,20,30,40$ and $50 \mu \mathrm{mol} / 1$ for CNE-2; 0, 5, 10, 30, 70, 100 and $200 \mu \mathrm{mol} / \mathrm{l}$ for 6-10B; Santa Cruz Biotechnology). Cells were incubated under standard culture conditions for $48 \mathrm{~h}$, and viable cells were assessed using the CCK- 8 assay kit. 


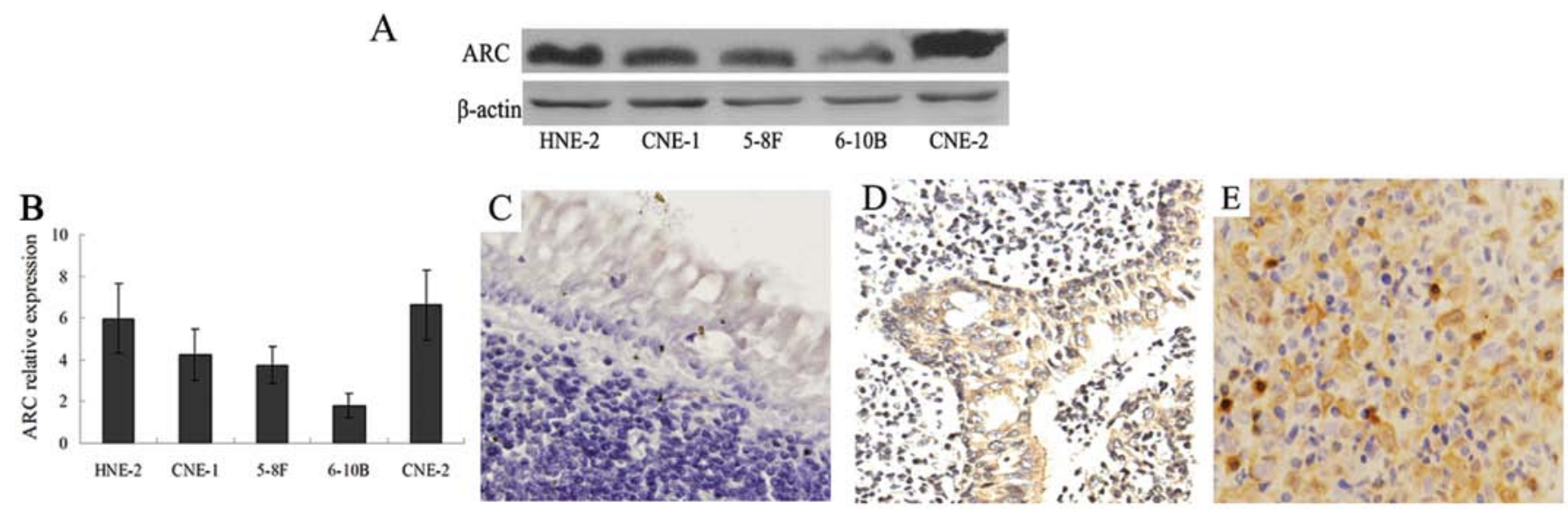

Figure 1. ARC protein levels in nasopharyngeal carcinoma (NPC) cell lines and NPC tissue. (A) Western blot analysis. Protein from the indicated nasopharyngeal carcinoma cell lines were immunoblotted for ARC and $\beta$-actin. (B) Quantitation of ARC abundance normalized to that of $\beta$-actin. (C) Immunohistochemistry of benign nasopharynx tissue showing undetectable ARC levels. (D) Immunohistochemistry of severe atypical hyperplasia tissue showing the presence of ARC. (E) Immunohistochemistry of NPC showing abundant ARC.

Table I. ARC in nasopharyngeal carcinoma (NPC).

\begin{tabular}{|c|c|c|c|c|}
\hline Classification & $\begin{array}{c}\text { Cytoplasmic ARC } \\
\text { immunostaining } \\
\text { positive }(\%) ; \mathrm{P}_{50}\left[\mathrm{P}_{25} ; \mathrm{P}_{75}\right]\end{array}$ & P-value & $\begin{array}{c}\text { Nuclear ARC } \\
\text { immunostaining } \\
\text { positive }(\%) ; \mathrm{P}_{50}\left[\mathrm{P}_{25} ; \mathrm{P}_{75}\right]\end{array}$ & P-value \\
\hline Benign nasopharyngeal tissue & $1 / 20 \quad(5.0) ; \quad 1[0 ; 1]$ & & $0 / 20 \quad(0.0) ; \quad 0[0 ; 1]$ & \\
\hline Severe atypical hyperplasia & $9 / 14(64.3) ; \quad 3[1.75 ; 4.25]$ & $\chi^{2}=42.446^{\mathrm{a}}$ & $1 / 14 \quad(7.2) ; 1.5[1 ; 2]$ & $\chi^{2}=34.025^{\mathrm{a}}$ \\
\hline NPC & $72 / 82(87.8) ; \quad 4[3 ; 5]$ & $<0.001$ & $14 / 82(17.1) ; 2[1 ; 2]$ & $<0.001$ \\
\hline \multicolumn{5}{|l|}{ Gender (NPC) } \\
\hline Male & $41 / 47(87.2) ; \quad 4[3 ; 5]$ & $\mathrm{Z}=-0.724^{\mathrm{b}}$ & $8 / 47(17.0) ; \quad 2[1 ; 2]$ & $Z=-0.650^{b}$ \\
\hline Female & $31 / 35(88.6) ; 4[3 ; 5]$ & 0.469 & $6 / 35(17.1) ; \quad 2[1 ; 2]$ & 0.516 \\
\hline \multicolumn{5}{|l|}{ Primary tumor $(\mathrm{T})$ stage (NPC) } \\
\hline $\mathrm{T}_{1-2}$ & $33 / 43 \quad(76.7) ; 3[3 ; 5]$ & $Z=-5.145^{b}$ & $0 / 43 \quad(0.0) ; \quad 2[1 ; 2]$ & $Z=-5.829^{b}$ \\
\hline $\mathrm{T}_{3-4}$ & $39 / 39(100.0) ; 5[4 ; 6]$ & $<0.001$ & $14 / 39(35.9) ; 2[2 ; 4]$ & $<0.001$ \\
\hline \multicolumn{5}{|l|}{ Lymph node metastasis (NPC) } \\
\hline Negative & $21 / 26(80.8) ; \quad 4[3 ; 5]$ & $\mathrm{Z}=-1.289^{\mathrm{b}}$ & $6 / 26(23.1) ; \quad 2[2 ; 2]$ & $Z=-0.327^{b}$ \\
\hline Positive & $51 / 56(91.1) ; 5[3 ; 6]$ & 0.198 & $8 / 56(14.3) ; \quad 2[1 ; 2]$ & 0.743 \\
\hline
\end{tabular}

$\mathrm{P}_{50}$, fiftieth percentile (median); $\mathrm{P}_{25}$, twenty-fifth percentile value; $\mathrm{P}_{75}$, seventy-fifth percentile value. ${ }^{a}$ Kruskal-Wallis test; ${ }^{b}$ Mann-Whitney $\mathrm{U}$ test.

The half-maximal inhibitory concentration $\left(\mathrm{IC}_{50}\right)$ values were determined with SPSS 17.0 software.

Statistical analyses. All statistical analyses were carried out using SPSS for Windows version 17.0 (SPSS). Log-rank test and one-way analysis of variance (ANOVA) were used to analyze the significance of differences. All cell culture experiments were performed in triplicate. $\mathrm{P}<0.05$ was considered to indicate a statistically significant result.

\section{Results}

ARC protein is abundant in several NPC cell lines. Since ARC plays an important role in apoptosis escape and the development of cancers, we hypothesized that ARC is induced in NPC cell lines. To test this hypothesis, we assessed the abundance of ARC in 5 cell lines using western blotting (Fig. 1A). ARC was detectable in most cell lines but present to a variable extent. Quantitative analysis revealed that levels were highest in CNE-2 and lowest in 6-10B cells (Fig. 1B). ARC was also present in the other NPC cell lines including CNE-1, 5-8F and HNE-2.

ARC protein is increased in NPC tissues. Given the high ARC levels in several NPC cell lines, we assessed whether the ARC levels were increased in NPC tissues. We next carried out an immunohistochemical analysis of an independent group of 82 NPC, 14 severe atypical hyperplasia and 20 benign nasopharyngeal tissues. On a scale of 0-6, a score 0-2 was considered to be negative; a score 3-6 was considered to be positive. Quantitative analysis of data (Table I) revealed ARC staining in the cytoplasm of $87.8 \%$ of NPC, $64.3 \%$ of severe atypical 
A

\begin{tabular}{|c|c|c|c|}
\hline & $6-10 B$ & $\mathrm{CNE}$ & \\
\hline & $\overline{\text { Control }}$ ARC & miCtrl & miARC \\
\hline $\mathrm{ARC}$ & 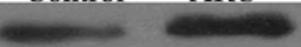 & 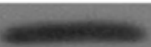 & 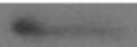 \\
\hline$\beta$-actin & $=$ & - & 20 \\
\hline
\end{tabular}
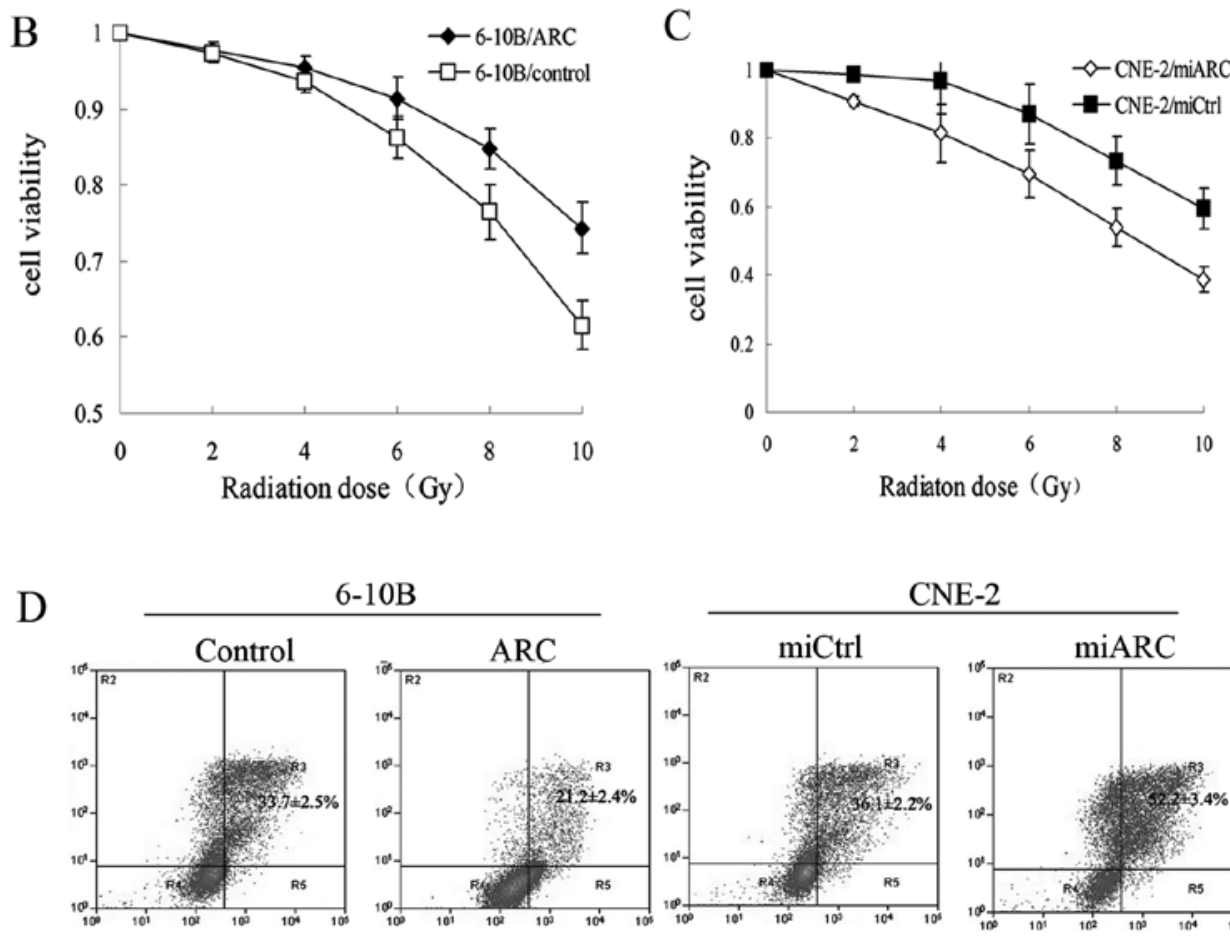

6-10B

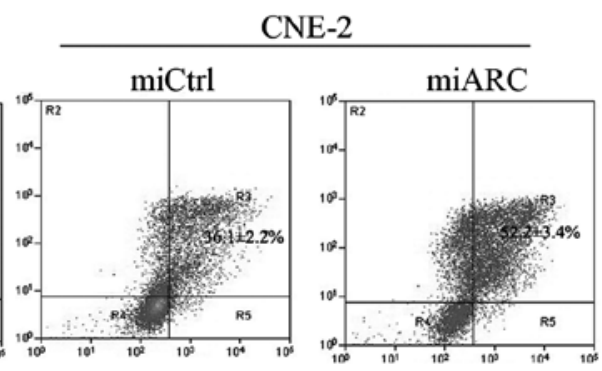

Figure 2. ARC induces radiation-resistance in NPC cells. (A) ARC expression in ARC-transfected 6-10B cells and ARC-silenced CNE-2 cells as determined by western blot analysis. (B) Cell viability of ARC-overexpressing or control 6-10B cells exposed to radiation. (C) Cell viability of miARC- or miCtrltransfected CNE-2 cells exposed to radiation. (D) The proportion of Annexin V-stained apoptotic cells in the ARC-overexpressing 6-10B cells and in the miARC-transfected CNE-2 cells and their control cells was determined by flow cytometric analysis at $72 \mathrm{~h}$ after irradiation with 8 Gy X-ray.

hyperplasia and only $5.0 \%$ of the benign nasopharyngeal tissues $(\mathrm{P}<0.001$; Table I). The median staining intensity of cytoplasmic ARC was 4 in NPC, 3 in atypical hyperplasia and only 1 in benign nasopharyngeal tissues $(\mathrm{P}<0.001$; Table I). ARC was present in the nucleus in $17.1 \%$ of NPC and in $7.2 \%$ of severe atypical hyperplasia tissues and ARC was not detected in benign nasopharyngeal tissue $(\mathrm{P}<0.001$; Table I). The median staining intensity of nuclear ARC was 2 in NPC and 1.5 in atypical hyperplasia tissues $(\mathrm{P}<0.001$, Table I). These data demonstrate that the abundance of ARC was increased in the cytoplasm of nearly all NPC tissues and a large part of the severe atypical hyperplasia tissues, but was present only rarely and at low levels in the benign nasopharyngeal tissues. Representative examples of the differential presence of ARC in different histopathological tissues are shown in Fig. 1C-E. In addition, we found that only a small portion of the NPC tissues contained nuclear ARC, although nuclear ARC was more prevalent in NPC than in the severe atypical hyperplasia and benign nasopharyngeal tissues.

We next explored the relationship between the clinicopathological parameters of NPC and the subcellular localization of ARC. The median intensity of cytoplasmic ARC staining was 4 in the male patients compared to 4 in the female patients ( $\mathrm{P}>0.05$; Table I), 5 in stage $\mathrm{T}_{3-4}$ tumors compared to 3 in stage
$\mathrm{T}_{1-2}$ tumors $(\mathrm{P}<0.001$; Table $\mathrm{I}), 4$ in the patients with lymph node metastasis compared to 4 in patients with no lymph node metastasis ( $\mathrm{P}>0.05$; Table I). The median intensity of nuclear ARC staining was 2 in male compared to 2 in female patients ( $\mathrm{P}>0.05$; Table $\mathrm{I}$ ), 2 in stage $\mathrm{T}_{3-4}$ tumors compared to 2 in stage $\mathrm{T}_{1-2}$ tumors $(\mathrm{P}<0.001$; Table $\mathrm{I}), 2$ in patients with lymph node metastasis compared to 2 in patients with no lymph node metastasis $(\mathrm{P}>0.05$; Table I). These data demonstrate that both cytoplasmic and nuclear ARC is present at a higher level in advanced NPC than in early-stage NPC. However, neither cytoplasmic nor nuclear ARC was associated with gender or lymph node metastasis.

Overexpression of ARC increases the resistance to $X$-radiation. Since ARC is known to inhibit apoptosis through extrinsic and intrinsic apoptotic pathways and presents at high levels in most NPC tissues, we hypothesized that this protein may contribute to radiation-resistance in NPC. To confirm this hypothesis, ARC-overexpressing 6-10B cells, ARC-silenced CNE-2 cells and their respective control cells (Fig. 2A) were exposed individually to $\mathrm{X}$-ray radiation (2-10 Gy). Cell viability was then examined after $96 \mathrm{~h}$. We found that cell viability in the ARC-overexpressing 6-10B cells was significantly increased at each indicated dose compared with the control cells 
A

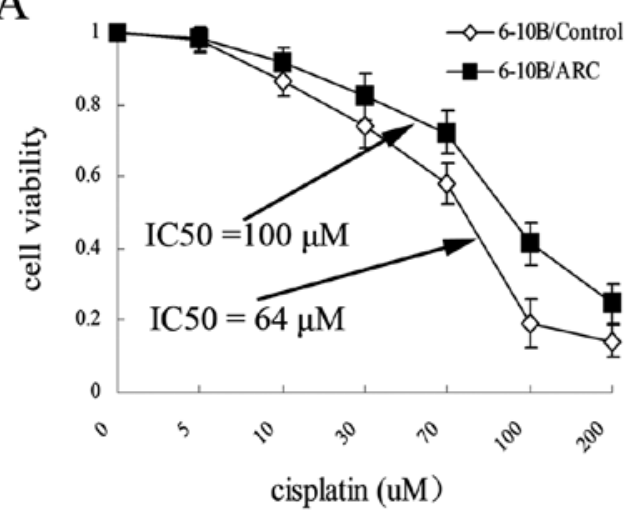

$\mathrm{C}$

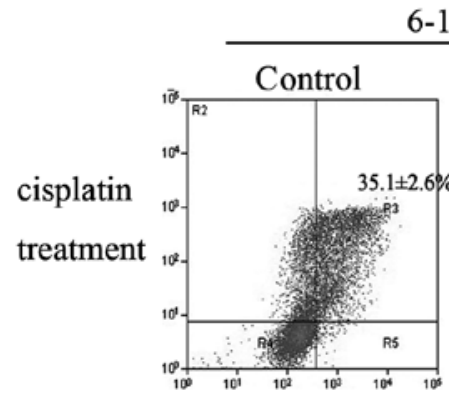

$6-10 \mathrm{~B}$

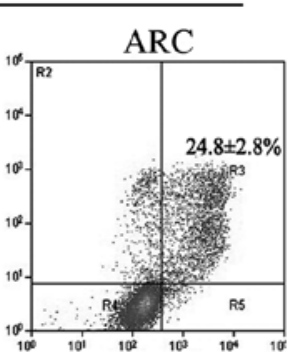

$\mathrm{B}$

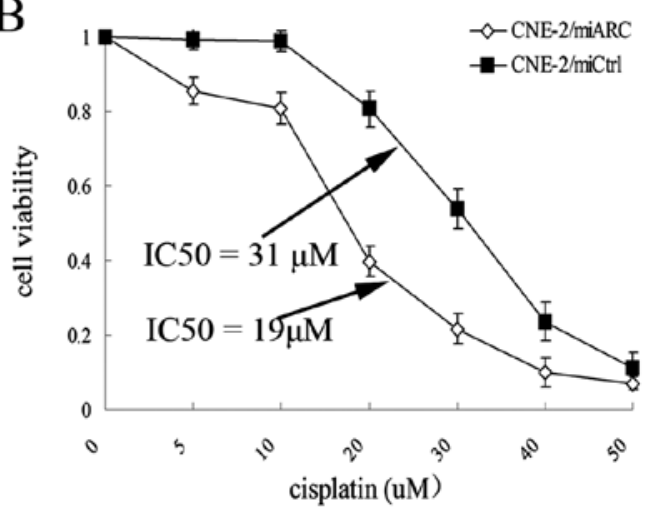

CNE-2

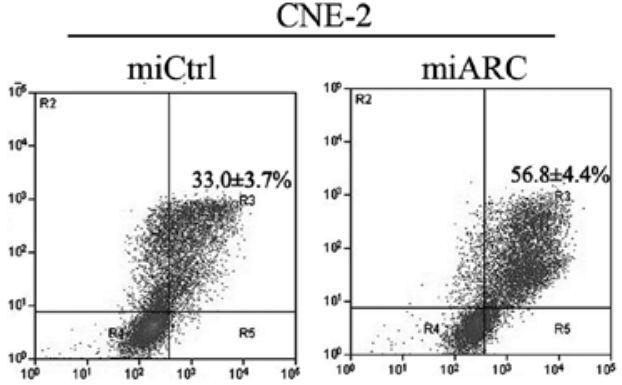

Figure 3. ARC induces chemotherapy-resistance in NPC cells. (A) Cell viability of ARC-overexpressing or control 6-10B cells following exposure to cisplatin. Overexpression of ARC in 6-10B cells increased the resistance to cisplatin. (B) Cell viability of miARC- or miCtrl-transfected CNE-2 cells following exposure to cisplatin. Knockdown of ARC in CNE-2 cells sensitized cells to cisplatin. (C) The proportion of Annexin V-stained apoptotic cells in ARC-overexpressing 6-10B cells and miARC-transfected CNE-2 cells and their control cells was determined by flow cytometric analysis at $48 \mathrm{~h}$ after cisplatin treatment.

(P<0.001; Fig. 2B). The ARC-transfected 6-10B cells were less sensitive to radiation than the control cells. Furthermore, the ARC-silenced CNE-2 cells exhibited decreased cell viability and increased sensitivity to radiation when compared to the control cells $(\mathrm{P}<0.001$; Fig. 2C).

ARC decreases $X$-radiation-induced apoptosis. We next investigated the effect of ARC on radiation-induced cell apoptosis. Cells were exposed to 8-Gy radiation and the proportion of apoptotic cells was assessed using the Annexin V-FITC apoptosis detection kit. Compared to the control cells, overexpression of ARC in 6-10B cells resulted in a lower proportion of apoptotic cells. The percentage of apoptotic cells in the ARC-overexpressing 6-10B cells was significantly decreased when compared to the controls $(21.2 \pm 2.4$ vs. $33.7 \pm 2.5 \%$, $\mathrm{P}<0.001)$. Similarly, when ARC was knocked down in CNE-2 cells, the number of apoptotic cells after radiation treatment was significantly increased when compared to the control cells (52.2 \pm 3.4 vs. $36.1 \pm 2.2 \%, \mathrm{P}<0.001)$ (Fig. 2D). These results indicate that $\mathrm{ARC}$ attenuates radiation-induced apoptosis in NPC cells.

Overexpression of ARC increases the resistance to cisplatin. As an apoptotic inhibitor, ARC may also imply a change of cell survival capacity against chemotherapeutic treatment. To test this hypothesis, the ARC-overexpressing 6-10B cells, ARC-silenced CNE-2 cells and their respective control cells were treated individually with cisplatin for $48 \mathrm{~h}$ and the cell viability was evaluated. When the cells were exposed to cisplatin, the viability of ARC-overexpressing 6-10B cells was significantly increased at each indicated dose compared with the control cells ( $\mathrm{P}<0.001$; Fig. 3A). The ARC-transfected 6 -10B cells were less sensitive to cisplatin $\left(\mathrm{IC}_{50}, 100 \mu \mathrm{M}\right)$ than the control cells $\left(\mathrm{IC}_{50}, 64 \mu \mathrm{M}\right)$. Furthermore, when ARC was silenced in CNE-2 cells, the CNE-2 cells showed an increased sensitivity to cisplatin $\left(\mathrm{IC}_{50}, 19 \mu \mathrm{M}\right)$ compared to the control cells $\left(\mathrm{IC}_{50}, 31 \mu \mathrm{M}\right)(\mathrm{P}<0.001$; Fig. 3B).

ARC decreases cisplatin-induced apoptosis. We also investigated the effect of ARC on cisplatin-induced cell apoptosis. Cells were exposed to cisplatin $\left(\mathrm{IC}_{30}\right)$ for $48 \mathrm{~h}$ and the proportion of apoptotic cells was assessed using the Annexin V-FITC apoptosis detection kit. Compared to the control cells, overexpression of ARC in 6-10B cells resulted in a lower proportion of apoptotic cells. The percentage of apoptotic cells in the ARC-overexpressing 6-10B cells was significantly decreased when compared to the controls $(24.8 \pm 2.8$ vs. $35.1 \pm 2.6 \%$, $\mathrm{P}<0.001)$. Consistent with the reduced viability, knockdown of ARC promoted cell apoptotic activity. The prercentage apoptotic cells after repression of ARC was increased compared to that observed in the cells without silencing of ARC $(56.8 \pm 4.4$ vs. $33.0 \pm 3.7 \%, \mathrm{P}<0.001$ ) (Fig. $3 \mathrm{C}$ ). These results indicate that ARC protects cells from cisplatin-induced apoptosis.

Exogenous ARC inhibits radiation and cisplatin-induced caspase-2 and caspase- 8 activation. To understand the mechanism(s) by which exogenous ARC protects NPC cells from radiation- or cisplatin-induced apoptosis, we detected 


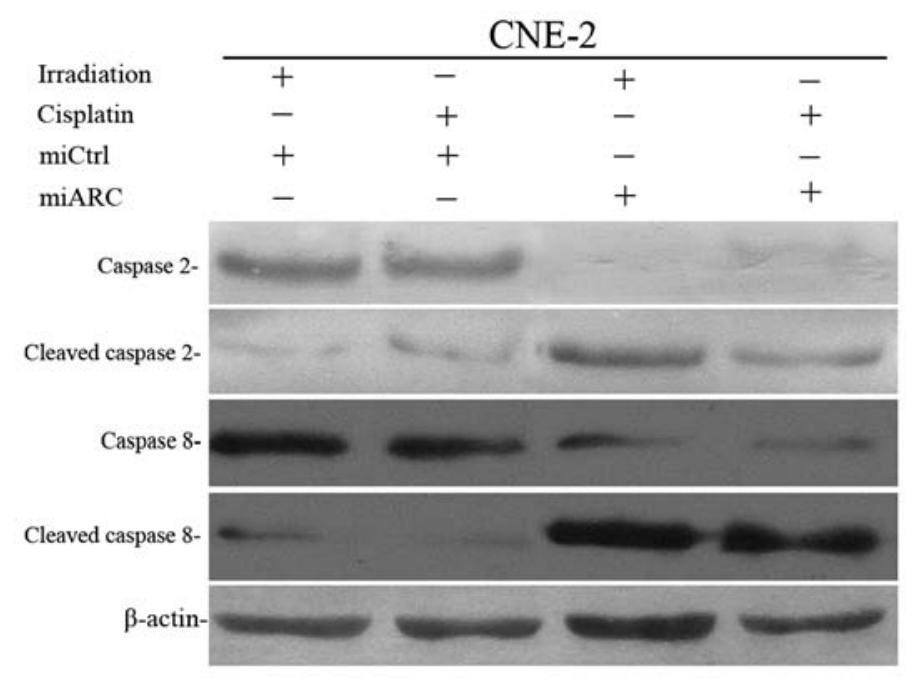

Figure 4. Endogenously expressed ARC contributes to the blockage of activation of caspase-2 and caspase-8. Whole-cell lysates were subjected to western blot analysis for the expression of caspase- 2 and caspase- 8 . The data shown are representative of three individual experiments.

caspase- 2 and caspase- 8 activation in the ARC-silenced CNE-2 cells after exposure to X-radiation or cisplatin. As shown in Fig. 4, miRNA knockdown of ARC enhances X-radiation- or cisplatin-induced caspase-2 and caspase- 8 activation.

\section{Discussion}

ARC is primarily expressed in terminally differentiated cells, such as heart, skeletal muscle and nerve cells $(4,12)$. Recent research suggests that ARC is highly expressed in a variety of non-terminally differentiated tissues and in human cancer cell lines, including those derived from breast, lung, pancreas, colon, cervix, prostate, kidney and lymph (13). Expression of ARC in primary epithelial-derived breast cancer and colon cancer was also higher than that in the respective benign epithelial tissues $(14,15)$, but the applicability of this finding to NPC remains unknown.

The present study assessed the expression of ARC in NPC cell lines and NPC tissues. Western blot analysis results demonstrated that ARC was present to a variable extent in several NPC cell lines. ARC level was high in CNE-2, HNE-2 and CNE-1 cells; in contrast, its level was low in $5-8 \mathrm{~F}$ and 6-10B cells. Because of the inherent limitations of cell lines, we assessed ARC levels in primary NPC, severe atypical hyperplasia and benign nasopharyngeal tissues by immunohistochemistry. We found that the amount of cytoplasmic ARC was increased in NPC and severe atypical hyperplasia but not in benign nasopharyngeal tissues. Moreover, cytoplasmic and nuclear ARC was increased in $\mathrm{T}_{3-4}$ stage NPC, which indicates that high expression of ARC provides a direct correlation with advanced local invasion. In addition, we found that only a small amount of nuclear ARC was expressed in NPC and severe atypical hyperplasia tissues in contrast to cytoplasmic ARC, which is similar to expression in human breast cancer (14). These results demonstrate that ARC may serve as a novel marker of NPC and suggest that it may be important in the pathogenesis of NPC.
Carcinogenesis often involves defects in apoptosis that is mediated by an increase in apoptotic inhibitors. It was previously found that multiform endogenous apoptosis inhibitors are significantly present at high levels in numerous types of cancers. For example, survivin, cIAP2 and XIAP which belong to the IAP family, were significantly more highly expressed in colon cancer, prostate cancer and leukemia, and high expression of these molecules indicated a poor prognosis (16-18). Similarly, Bcl-2 and Bcl-xl were highly expressed in leukemia, breast, colon, esophageal, pancreatic and uterine cancer (19-24). Thus, the abnormal expression of ARC may provide NPC cells with a survival mechanism that normally serves to protect cancer cell populations.

Given such strong evidence implicating the role of ARC in enhancing the malignant potential of NPC, in the present study, we investigated whether ARC also plays a direct role in mediating radiation- and chemo-resistance in NPC. Regarding radiotherapy, we showed that attenuation of ARC expression by miRNA treatment resulted in a decreased rate of cell viability as well as increased X-radiation-induced apoptosis in CNE-2 cells. In contrast, overexpression of ARC in 6-10B cells resulted in an increased rate of cell viability and decreased $\mathrm{X}$-radiation-induced apoptosis. Similarly, regarding chemotherapy, silencing of ARC resulted in a decreased rate of cell viability and increased apoptosis following cisplatin exposure in CNE-2 cells. In contrast, overexpression of ARC increased the rate of cell viability and decreased apoptosis following cisplatin exposure in 6-10B cells. Moreover, the mechanism(s) of ARC in the protection of NPC cells from X-radiation- or cisplatin-induced apoptosis was shown by miRNA knockdown of ARC in CNE-2 cells, which revealed that ARC suppressed the activation of caspase- 2 and caspase- 8 . Thus, ARC expression seems to be a marker of radiation- and chemo-resistance in vitro.

Radiation therapy has been thought to induce cell death mainly by apoptosis $(25,26)$. Similarly, cisplatin exerts its cytotoxic effect by inducing DNA damage and activating apoptosis (27). ARC, as an apoptosis inhibitor through medi- 
ating both the extrinsic and intrinsic pathways, is likely to suppress radiation- and cisplatin-induced cell apoptosis. ARC was found to protect against doxorubicin and $\gamma$-radiation in breast cancer cells, and inhibition of ARC promoted apoptosis and sensitized cytosine arabinoside-induced cell death in OCI-AML3 cells $(14,28)$.

Caspase- 8 and caspase- 2 are initiator caspases that are activated following various forms of genotoxic stress, including that induced by radiation and cisplatin (29-32). Caspase-8 plays an important role in transducing the apoptosis signal to downstream death effectors, including CD95, TRAIL-DR4 or TRAIL-DR5, which results in formation of the DISC (33). Caspase-2 possesses a caspase recruitment domain (CARD) that enables it to interact with other CARD carrying proteins such as TRAF-2 and PIDD (p53 induced protein with a death domain), which eventually leads to activation of Bid and mitochondrial injury $(34,35)$. ARC can interact with caspase- 2 and caspase- 8 and inhibit their activation to protect against cell death.

In summary, the present study demonstrated that ARC is expressed at high levels in NPC cell lines. Moreover, abundant ARC was present in most NPC and severe atypical hyperplasia tissues, but not in benign nasopharyngeal tissues. Furthermore, we demonstrated the function of ARC in exerting X-radiation resistance and cisplatin resistance by inhibiting apoptosis through blocking the activation of caspase- 2 and caspase- 8 .

\section{Acknowledgements}

The present study was supported by the Natural Science Foundation of China (30672296).

\section{References}

1. Vaux DL and Strasser A: The molecular biology of apoptosis Proc Natl Acad Sci USA 93: 2239-2244, 1996.

2. Thompson CB: Apoptosis in the pathogenesis and treatment of disease. Science 267: 1456-1462, 1995.

3. Nam YJ, Mani K, Ashton AW, et al: Inhibition of both the extrinsic and intrinsic death pathways through nonhomotypic death-fold interactions. Mol Cell 15: 901-912, 2004.

4. Koseki T, Inohara N, Chen S, et al: ARC, an inhibitor of apoptosis expressed in skeletal muscle and heart that interacts selectively with caspases. Proc Natl Acad Sci USA 95: 5156-5160, 1998.

5. Gustafsson AB, Tsai JG, Logue SE, et al: Apoptosis repressor with caspase recruitment domain protects against cell death by interfering with Bax activation. J Biol Chem 279: 21233-21238, 2004

6. Foo RS, Nam YJ, Ostreicher MJ, et al: Regulation of p53 tetramerization and nuclear export by ARC. Proc Natl Acad Sci USA 104: 20826-20831, 2007.

7. Ekhterae D, Lin Z, Lundberg MS, et al: ARC inhibits cytochrome $\mathrm{c}$ release from mitochondria and protects against hypoxia-induced apoptosis in heart-derived H9c2 cells. Circ Res 85: e70-e77, 1999.

8. Neuss M, Monticone R, Lundberg MS, et al: The apoptotic regulatory protein ARC (apoptosis repressor with caspase recruitment domain) prevents oxidant stress-mediated cell death by preserving mitochondrial function. J Biol Chem 276 : 33915-33922, 2001.

9. Donath S, Li P, Willenbockel C, et al: Apoptosis repressor with caspase recruitment domain is required for cardioprotection in response to biomechanical and ischemic stress. Circulation 113: 1203-1212, 2006.

10. Wu P, Zhang H, Qi L, et al: Identification of ERp29 as a biomarker for predicting nasopharyngeal carcinoma response to radiotherapy. Oncol Rep 27: 987-994, 2012.

11. Qi L, Wu P, Zhang X, et al: Inhibiting ERp29 expression enhances radiosensitivity in human nasopharyngeal carcinoma cell lines. Med Oncol 29: 721-728, 2012.
12. Geertman R, McMahon A and Sabban EL: Cloning and characterization of cDNAs for novel proteins with glutamic acid-proline dipeptide tandem repeats. Biochim Biophys Acta 1306: 147-152, 1996.

13. Wang M, Qanungo S, Crow MT, et al: Apoptosis repressor with caspase recruitment domain (ARC) is expressed in cancer cells and localizes to nuclei. FEBS Lett 579: 2411-2415, 2005.

14. Mercier I, Vuolo M, Madan R, et al: ARC, an apoptosis suppressor limited to terminally differentiated cells, is induced in human breast cancer and confers chemo- and radiation-resistance. Cell Death Differ 12: 682-686, 2005.

15. Mercier I, Vuolo M, Jasmin JF, et al: ARC (apoptosis repressor with caspase recruitment domain) is a novel marker of human colon cancer. Cell Cycle 7: 1640-1647, 2008.

16. Sarela AI, Macadam RC, Farmery SM, et al: Expression of the antiapoptosis gene, survivin, predicts death from recurrent colorectal carcinoma. Gut 46: 645-650, 2000.

17. Krajewska M, Krajewski S, Banares S, et al: Elevated expression of inhibitor of apoptosis proteins in prostate cancer. Clin Cancer Res 9: 4914-4925, 2003.

18. Tamm I, Kornblau SM, Segall H, et al: Expression and prognostic significance of IAP-family genes in human cancers and myeloid leukemias. Clin Cancer Res 6: 1796-1803, 2000.

19. Hanada M, Delia D, Aiello A, et al: bcl-2 gene hypomethylation and high-level expression in B-cell chronic lymphocytic leukemia. Blood 82: 1820-1828, 1993.

20. Olopade OI, Adeyanju MO, Safa AR, et al: Overexpression of BCL-x protein in primary breast cancer is associated with high tumor grade and nodal metastases. Cancer J Sci Am 3: 230-237, 1997.

21. Friess H, Lu Z, Andren-Sandberg A, et al: Moderate activation of the apoptosis inhibitor bcl-Xl worsens the prognosis in pancreatic cancer. Ann Surg 228: 780-787, 1998.

22. Marone M, Scambia G, Mozzetti S, et al: bcl-2, bax, bcl-XL and bcl-XS expression in normal and neoplastic ovarian tissues. Clin Cancer Res 4: 517-524, 1998

23. Biroccio A, Benassi B, D'Agnano I, et al: c-Myb and Bcl-x overexpression predicts poor prognosis in colorectal cancer: clinical and experimental findings. Am J Pathol 158: 1289-1299, 2001.

24. Takayama T, Nagao M, Sawada H, et al: Bcl-X expression in esophageal squamous cell carcinoma: association with tumor progression and prognosis. J Surg Oncol 78: 116-123, 2001.

25. Verma YK, Raghav PK, Raj HG, et al: Enhanced heterodimerization of Bax by Bcl-2 mutants improves irradiated cell survival. Apoptosis 18: 212-225, 2013

26. Huang S, Benavente S, Armstrong EA, et al: p53 modulates acquired resistance to EGFR inhibitors and radiation. Cancer Res 71: 7071-7079, 2011.

27. Gong JG, Costanzo A, Yang HQ, et al: The tyrosine kinase c-Abl regulates $\mathrm{p} 73$ in apoptotic response to cisplatin-induced DNA damage. Nature 399: 806-809, 1999.

28. Carter BZ, Qiu YH,Zhang N, et al: Expression of ARC (apoptosis repressor with caspase recruitment domain), an antiapoptotic protein, is strongly prognostic in AML. Blood 117: 780-787, 2011.

29. Chen $T$, Chen $M$ and Chen J: Ionizing radiation potentiates dihydroartemisinin-induced apoptosis of A549 cells via a caspase-8-dependent pathway. PLoS One 8: e59827, 2013.

30. Paul I, Chacko AD, Stasik I, et al: Acquired differential regulation of caspase-8 in cisplatin-resistant non-small-cell lung cancer. Cell Death Dis: e449, 2012.

31. Cao X, Bennett RL and May WS: c-Myc and caspase-2 are involved in activating Bax during cytotoxic drug-induced apoptosis. J Biol Chem 283: 14490-14496, 2008.

32. Hanoux V, Pairault C, Bakalska M, et al: Caspase-2 involvement during ionizing radiation-induced oocyte death in the mouse ovary. Cell Death Differ 14: 671-681, 2007.

33. Wang S and El-Deiry WS: TRAIL and apoptosis induction by TNF-family death receptors. Oncogene 22: 8628-8633, 2003.

34. Cuenin S, Tinel A, Janssens S, et al: p53-induced protein with a death domain (PIDD) isoforms differentially activate nuclear factor-kappaB and caspase-2 in response to genotoxic stress. Oncogene 27: 387-396, 2008.

35. Lassus P, Opitz-Araya X and Lazebnik Y: Requirement for caspase-2 in stress-induced apoptosis before mitochondrial permeabilization. Science 297: 1352-1354, 2002. 